\title{
On the Capacity of Wi-Fi System in TV White Space with Aggregate Interference Constraint
}

\author{
Yanpeng Yang, Lei Shi, and Jens Zander \\ KTH Royal Institute of Technology, Wireless@KTH, Stockholm, Sweden \\ E-mail: yanpeng@kth.se, 1shi@kth.se,jenz@kth.se
}

\begin{abstract}
In this paper, we present a quantitative assessment of the performance of a Wi-Fi like system in TV white space and compare it with the traditional Wi-Fi system in the ISM band. Particular emphasis is given to the constraint on the aggregate secondary interference on $\mathrm{TV}$ reception, which is regarded as the premise of the link throughput analysis that followed. Numerical evaluation is performed over different scenarios where the TV receive signal strength, number of unoccupied $\mathrm{TV}$ channels, user density are varied. Our results show that the primary interference constraint has only significant impact on the secondary system performance in rural scenario with low $T V$ receive signal strength. In other cases, where higher transmit power does not guarantee better performance, the capacity of the system is limited by the secondary self-interference or collisions rather than the interference constraint. Therefore, efficient TV White Space utilization can be achieved by properly setting the secondary transmit power adaptive to different environments.
\end{abstract}

Index Terms-TV White Space, secondary spectrum reuse, aggregate interference, adjacent channel interference, geo-location database.

\section{INTRODUCTION}

In recent years, the growing demand for high data-rate wireless services has led to the so-called 'spectrum shortage' for wireless broadband access. As predicated by Wireless Broadband Alliance, most parts of Europe will experience congestion in the bands for shared access in the next five years [1]. On the other hand, results from the latest studies indicate that a considerable amount of spectrum is under-utilized due to the traditional regulatory framework and the limit of exclusive licensing. To increase the utilization of these frequency bands, the concept of secondary access has been proposed to allow them to be accessed by other users on a secondary basis, with the condition that the quality of service (QoS) of the primary users (PU) must be protected. One of the popular candidate bands to be opened up for secondary access is VHF/UHF bands for TV broadcasting, which is known as TV white space (TVWS). It has the potential to enable high quality outdoor-toindoor or indoor-to-outdoor wireless broadband coverage and become a significant complement to the existing spectrum of wireless communication [2].

The Federal Communications Commission (FCC) in the USA [3], Ofcom in the UK [4], CEPT in Europe [5] have all started to develop frameworks for the secondary usage of the TVWS band. In [6], the availability of TVWS in the 470$790 \mathrm{MHz}$ VHF/UHF band has been quantified for a number of European countries. Recently there has been great interest in using TVWS for providing wireless communications like WiFi or femto-cell network. The major challenges for operating Wi-Fi system in TVWS are the restrictions on permissible transmit power and number of available channels as the TV reception must be protected from any harmful secondary interference. Unlike the former research focus on the operation of autonomous secondary user (SU) based on sensing [7], a new geo-location database method is proposed in [8], where a SU would only need to report its location to the database and in return receive information regarding the spectrum availability and associated constraints. [9] extends the regulation framework to a multiple secondary user case considering the random deployment of SUs, TV receiver antenna directivity, and the cumulative effect of adjacent channel interference (ACI). Nevertheless, the TVWS opportunity is only evaluated in terms of permissible transmit power of different secondary users.

The architecture and capacity of a Wi-Fi like secondary system in TVWS are studied in [10]. The authors here describe an approach for providing wireless broadband access in urban areas by deploying SUs in TVWS with an inside-out network build strategy. Similarly, a performance analysis of a Wi-Fi like network is presented in [11]. Both of the results demonstrate the advantage of TVWS in the coverage sense. However, without channel aggregation, the Wi-Fi like secondary system in TVWS shows no advantage in throughput against the higher channel bandwidth available to Wi-Fi system in the ISM band. Moreover, these studies neglect the impact from the SUs to the PU - the TV system. The transmit power of the SUs were set to certain levels without any explicit limitation or consideration of protecting TV reception. Therefore, the performance of the secondary Wi-Fi system may have been overestimated.

This paper instead combines TV protection and the interaction among the secondary users in the analysis in order to draw a more complete picture of the performance of the secondary system in the TV band. The average throughput of a Wi-Fi like secondary system with CSMA/CA protocol deployed in the TV band is compared to that of the traditional Wi-Fi in the ISM band with various assumptions on the environmental conditions, such as different numbers of available channels, TV signal strength and SU densities. As prerequisite for operating in the TV band, the secondary transmit power is always restricted, while no primary constraint is applied to 


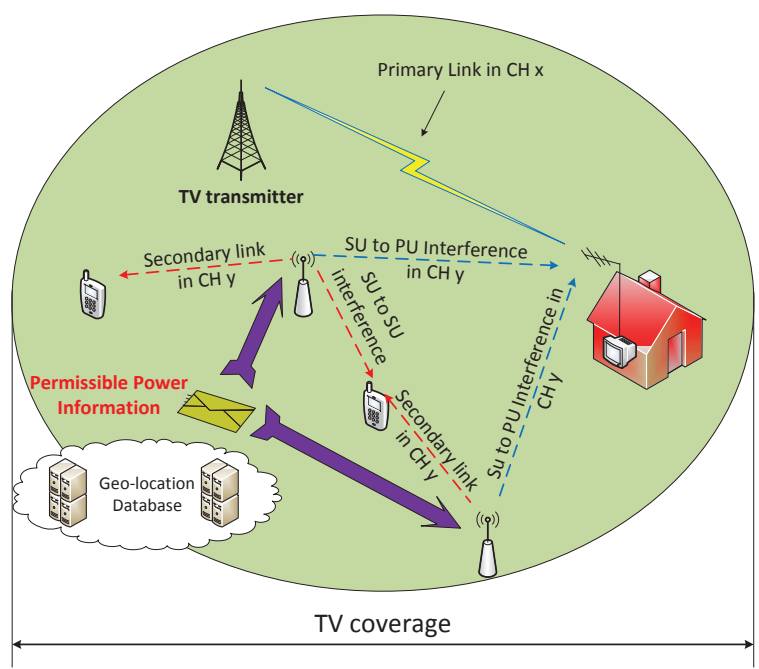

Fig. 1: Geo-location database coexistence model for secondary access in TVWS.

the Wi-Fi in the ISM band ${ }^{1}$. Furthermore, we aim to identify whether the capacity of the secondary system is truly limited by the TV protection constraint in different environment settings. Our simulation model considers the aggregate secondary interference to the TV receiver, SU-to-SU interference and CSMA/CA contention effects, so that the TV protection is not overlooked and the secondary system performance is not exaggerated.

The remainder of this paper is organized as follow: Section II provides a brief overview of the studied scenario. The system model is explained in Section III. Then we describe the simulation models in Section IV. In Section V, we present and analyze the numerical results. Finally, the conclusions are discussed in Section VI.

\section{Coexistence Model}

\section{A. TV Coverage and Protection Model}

A general Primary-Secondary user coexistence model is shown in Fig. 1. We assume that the primary links are operating in channel $x$ in the TV coverage area. Then the SUs are allowed to use the channels other than channel $x$ inside the coverage of the primary TV transmitter.

The measure for TV coverage quality is the location probability, defined as the chance of successful TV reception. Unsuccessful TV reception is termed outage, either due to the TV signal fading or interferences. The location probability without secondary interference is designated $q_{1}$

$$
q_{1}^{i}=\operatorname{Pr}\left\{P_{t v}^{i} \geq P_{t v}^{\min }+\gamma_{t v} I_{t v}^{i}\right\},
$$

where $P_{t v}^{i}$ and $P_{t v}^{\min }$ denote the receive power of the $i$ th TV and the minimum TV receiver sensitivity level. $\gamma_{t v}$ represents the required minimum ratio between the TV signal and TV

\footnotetext{
${ }^{1}$ Besides, we assume all Wi-Fi like devices have a physical transmit power constraint which ranges from $0 \mathrm{dBm}$ to $30 \mathrm{dBm}$.
}

self-interference and $I_{t v}^{i}$ is the received TV self-interference power from other TV transmitters. The coverage area is defined by $q_{1}^{i} \geq q^{*}$, with $q^{*}$ being the minimum required location probability defined by the regulator. To promise a good TV coverage, $q^{*}$ is usually set to at least $95 \%$.

When a SU is introduced, the geo-location database can determine the permissible power $P_{s u}^{j, y}$ for the $j$ th SU transmitting in TVWS channel $y$. The reduced location probability $q_{2}^{i, x}$ under secondary interference should be no less than $q^{* *}$ :

$$
q_{2}^{i, x}=\operatorname{Pr}\left\{P_{t v}^{i, x} \geq P_{t v}^{m i n}+\gamma_{t v} I_{t v}^{x}+I_{s u, a}^{i, x}\right\} \geq q^{* *},
$$

where $I_{s u, a}^{i, x}$ is the cumulative effect of the multi-channel aggregate secondary interference on the $i$ th TV receiver on the $x$ th TV channel. $q^{* *}$ represents a lower minimum location probability requirement under the secondary interference. Note that the secondary users contributing to the aggregate interference may be transmitting on different channels, as previous study [12] has confirmed. This effect is most noticeable when the SU is transmitting at close distance from the TV receiver. An analytical approximation is proposed in [9] to model the accumulative effect as

$$
I_{s u, a}^{x}=\sum_{n=1}^{N} \frac{\gamma_{s u}\left(\Delta f_{x-y_{n}}\right)}{\gamma_{s u}(0)} P_{s u}^{y_{n}} g_{n},
$$

where $\gamma_{s u}(\Delta f)$ is the TV protection ratio with frequency offset of $\Delta f . g_{n}$ denotes the coupling gain of the $n$th interfering link from adjacent channels $y_{n} . N$ is the total number of active SUs. The TV-to-TV interference is neglected in this paper due to its small value compared with this cumulative secondary interference.

\section{B. Geo-location Database}

According to its definition, the protection requirement should be applied to each TV receiver. However, the exact location of the TV receiver cannot be detected in most cases. Therefore, the regulator has instead discretized the coverage area into small area elements, denoted as 'pixel'. The related information, such as TV coverage quality, terrain elevation, user density, unoccupied TV channel numbers, etc., are stored for each pixel in the geo-location database. Based on this information, the permissible secondary transmit power $P_{s u}$ is obtained by Monte carlo simulation for each SU in pixel following the procedures outlined in [5] and [9]. In pixel $i$, all TV receivers are assumed to have approximately the same received TV signal strength $P_{t v}^{i}$. Any randomly selected TV from one pixel would experience statistically the same level of interference [9].

\section{Secondary System Model}

As mentioned earlier, we consider a Wi-Fi like secondary system with short range and low transmit power following CSMA/CA MAC protocol. Adjacent channel aggregations are allowed according to existing proposals. Given the continuity of the unoccupied TV channels, up to eight TV channels 
of $8 \mathrm{MHz}$ are aggregated in our work. One SU access point (AP) is assumed to be randomly deployed inside each house/apartment, and the users (UE) are randomly deployed within the coverage radius of the AP, which can be either indoors or outdoors. As the worst case assumption, we consider the SU-PU interfering links between outdoor SUs and the rooftop TV antenna in rural cases and the link between indoor SUs to the set-top TV antenna in urban cases. The link geometry is illustrated in Fig. 2. Note that co-channel interference (CCI) is not considered. As shown in [13], low power SU transmitting at below clutter height only causes negligible CCI as compared to the ACI inflicted on the TV receivers close by.

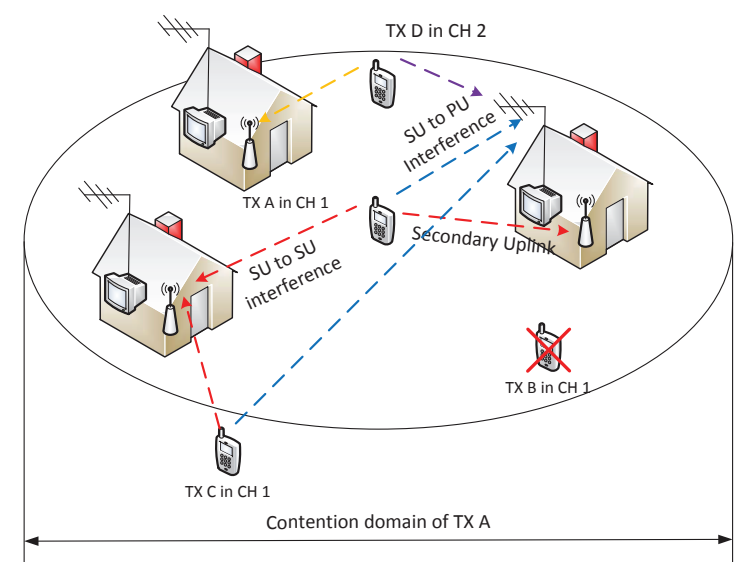

(a) Rural uplink scenario

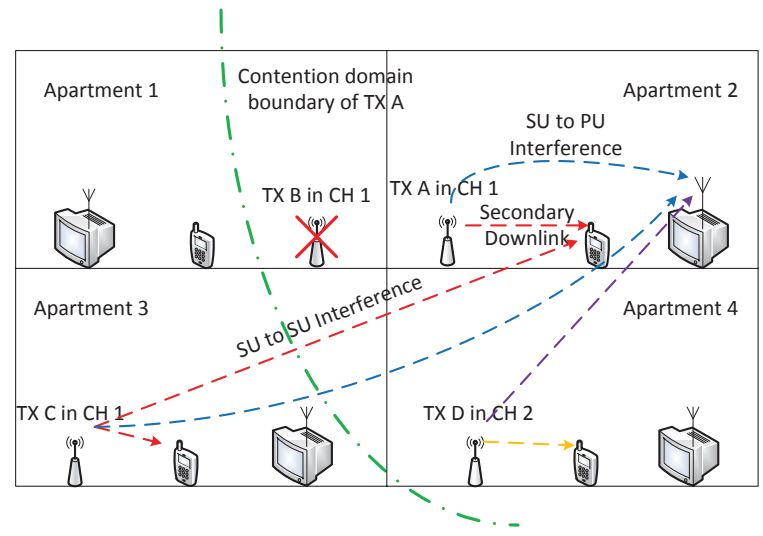

(b) Urban downlink scenario

Fig. 2: Link geometry and contention domain of secondary transmitters.

The SUs can transmit on any unoccupied TV channel whereas CSMA/CA protocol is applied to avoid collisions among SUs transmitting on the same channel. Given the detection threshold, the SU transmit power and the propagation environment, each SU with active traffic load is associated with a contention domain centered at itself. CSMA/CA protocol ensures that at any given time slot, there is at most one (randomly chosen) active secondary link within each contention domain.
To evaluate the performance of the secondary system, we define the average throughput of a secondary link as:

$$
\mathrm{TP}_{a v g}=\frac{1}{T} \frac{1}{N_{t o t}} \sum_{t=1}^{T} \sum_{n=1}^{N_{t}} \mathrm{TP}_{n}^{t},
$$

where $\mathrm{T}$ is the total simulation time slots, $N_{\text {tot }}$ is the total number of deployed SUs, $N_{t}$ is the number of active SUs transmitting without collision at time slot $t$. $\mathrm{TP}_{n}^{t}$ is the throughput of the $n$th active link obtained by Shannon formula ${ }^{2}$ :

$$
\begin{aligned}
\mathrm{TP}_{n}^{t} & =B W_{n} \log _{2}\left(1+S I N R_{n}\right) \\
& =B W_{n} \log _{2}\left(1+\frac{P_{s u, i}^{y} g_{n, n}}{N_{0}+\gamma_{p u} I_{p u}+\sum_{m \in \mathbf{A}_{n}^{y}} P_{s u, i}^{y} g_{m, n}}\right),
\end{aligned}
$$

where $g_{n, n}$ denotes the path loss between the $n$th transmitting SU and its receiver. $\gamma_{p u} I_{p u}$ represents the interference from PU and $N_{0}$ is the noise. As presented in [11], we define $\mathbf{A}_{n}^{y}$ as the set of SUs operating on channel $y$ outside the contention domain of the $n$th transmitting SU. Due to CSMA/CA protocol, these SUs are the ones causing co-channel interference to the $n$th receiving $\mathrm{SU}$ as shown in (5).

Although $P_{s u}$ can be set as much as it is permitted according to (2), it does not necessarily lead to the best performance. Because higher $P_{s u}$ may promise high SINR and consequently higher link throughput, but a larger contention domain also reduces the number of simultaneously active links due to collision avoidance. Thus, an optimal transmit power $P_{s u}^{o p t}$ is chosen by exhaustive search in simulation such that $\mathrm{TP}_{a v g}$ is maximized:

$$
P_{s u}^{o p t}=\underset{P_{s u}<P_{s u}^{*}}{\arg \max } \operatorname{TP}_{a v g}\left(P_{s u}\right),
$$

where $P_{s u}^{*}$ denotes the SU maximum permissible transmit power due to either physical limitation or primary protection constraint.

\section{Simulation Model}

\section{A. Simulation Procedure}

In order to reduce the boundary effect during the simulation, the SUs and TV receivers are deployed in a study area consisting of 3-by-3 pixels ${ }^{3}$, and only the SU links in the center pixel are used for throughput analysis. Without loss of generality, the pixel is assumed to be centered at a random chosen TV receiver to simplify the calculation of the aggregate secondary interference, which is supposed to be statistically identical for any $\mathrm{TV}$ in the same pixel. Note that, due to collision avoidance, not all deployed SUs would be active

\footnotetext{
${ }^{2}$ Auto-rate function is not applied for calculating throughput because SINR in our paper is sometimes significantly larger than the existing reference value in the table.

${ }^{3}$ The size of the studied area is chosen such that it is larger than the dominant interference region [9], where more than $99.5 \%$ of the aggregate ACI is originated.
} 
at the same time, thus we assume the geo-location database has integrated the CSMA/CA protocol into the Monte Carlo simulation process to determine the permissible transmit power such that the TV receiver location probability is no less than $94 \%$. Finally the optimal transmit power is obtained by maximizing the average link throughput by an iterative process while ensuring the TV protection.

\section{B. Scenario Model}

We consider two scenarios in this paper: rural and urban. In the rural scenario, small houses with roof-top antennas are randomly deployed in the pixels using a homogeneous Poisson point process with density $\lambda$. One set of secondary AP and UE is deployed for each house. To satisfy the worst interfering case, we consider a secondary uplink transmission (Fig. 2a). Edge performance is evaluated for a coverage distance of 50 meters. ITU-R P.1411 propagation models between terminals located over and below roof-top height [14] are adopted for interfering and transmission link respectively. In the urban scenario, five buildings of 7-floors are deployed regularly in each pixel. The user density is controlled through the density in each floor which contains several apartments. A downlink transmission (Fig. 2b) with a coverage distance up to 10 meters is considered in this scenario. ITU-R P.1238 propagation models [15] and Log-distance path loss model are used for transmissions inside the building and between different buildings respectively. Regarding the Wi-Fi system in the ISM band, an IEEE $802.11 \mathrm{~g}$ network with 3 nonoverlapping $20 \mathrm{MHz}$ channels operating in $2.4 \mathrm{GHz}$ is set according to [16].

\section{Simulation Parameter}

Monte Carlo simulations are performed to examine the impact of the environmental parameters on the system performance, including TV signal strength, user density and unoccupied TV channel number. Considering TV signal shadow fading standard deviation of $5.6 \mathrm{~dB}$, the minimum TV receive signal strength $P_{t v}^{i}$ should be at least $-71 \mathrm{dBm}$ to guarantee $q^{*} \geq 95 \%$. Thus the worst TV signal quality studied in our paper is $P_{t v}^{i}=-71 \mathrm{dBm}$. The value of user density and unoccupied TV channel number are set based on reference [18] and [6]. The parameters for the two scenarios are summarized in Table I.

\section{Numerical Results AND ANALysis}

\section{A. Impact of TV Signal Strength}

In Fig. 3, the impact of TV signal strength on average throughput is presented in both scenarios. In the rural scenario, the secondary user average throughput increases significantly as the TV signal quality improves. Nevertheless, there is an upper bound in throughput when the TV signal quality reaches certain level. This is illustrated in Fig. 4, which shows the relation between maximum permissible transmit power and optimal power selection. At low TV signal quality, the optimal power always selects the maximum permissible power because the secondary system is still noise limited. However, when the TV signal quality is good enough, the secondary system refrains from increasing its transmit power as it would result in a large contention domain and lower the throughput due to collision avoidance.

On the other hand, the TV signal quality has almost no effect on the secondary system performance in the urban scenario. It implies that the optimal transmit power of TVWS system could be achieved even with very low TV signal strength. The reason is twofold: firstly, the denser deployment in the urban scenario means a higher chance of collision; secondly, the shorter coverage distance requires less transmit power to achieve a reasonable SINR level.

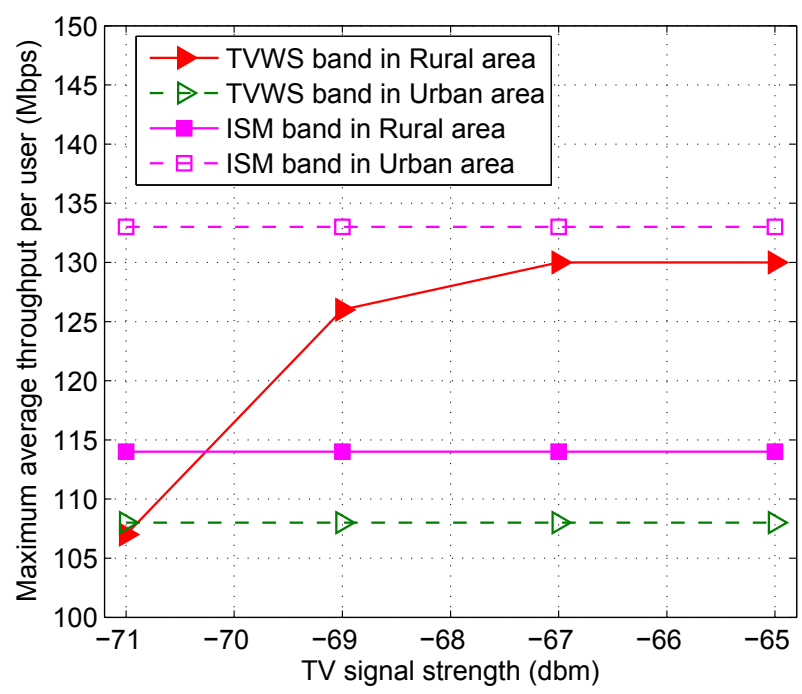

Fig. 3: Maximum average throughput versus TV signal strength. $\lambda=30 \mathrm{SUs} / \mathrm{km}^{2}$ in rural, $3000 \mathrm{SUs} / \mathrm{km}^{2}$ in urban, 2 channel aggregation, unoccupied TV channel number $=8$.

\section{B. Impact of Channel Aggregation}

The effect of different numbers of channel aggregation is illustrated in Fig. 5. Generally speaking, having a wider channel bandwidth results in a better throughput even though it means more SUs transmitting on the same channel and an increased risk of collision. Although a more realistic link throughput model, e.g., with lower bandwidth efficiency, may limit the advantage of having a wider channel bandwidth, the general trends would remain similar. With channel aggregation of four or more $8 \mathrm{MHz}$ channels, the secondary system can outperform the traditional Wi-Fi with $20 \mathrm{MHz}$ in the ISM band.

\section{Impact of TV Channel Availability}

Fig. 6 shows the impact of unoccupied TV channel number. As expected, having more available channels generally leads to a higher throughput. However, a somewhat surprising phenomenon is that the throughput actually declines when a large number of TV channels are available but the TV signal is weak. This is because a large number of available channels 
TABLE I: Simulation Parameter

\begin{tabular}{|c|c|c|}
\hline Parameter & Rural & Urban \\
\hline TV receiver sensitivity & $-80.6 \mathrm{dBm}$ & $-80.6 \mathrm{dBm}$ \\
TV signal standard deviation & $5.6 \mathrm{~dB}$ & $5.6 \mathrm{~dB}$ \\
TV receiver antenna directivity & Defined by ITU-R BT419-3 [17] & Omni-direction \\
TV antenna gain & $10 \mathrm{dBi}$ & $0 \mathrm{dBi}$ \\
TV protection ratio $\gamma_{s u}$ & $-37 \mathrm{~dB}$ & $-37 \mathrm{~dB}$ \\
Location Probability Threshold $q^{* *}$ & 0.94 & 0.94 \\
Unoccupied TV channel number & $4-32$ & $4-32$ \\
Pixel size & $1 \mathrm{~km} \times 1 \mathrm{~km}$ & $250 \mathrm{~m} \times 250 \mathrm{~m}$ \\
House/Building size & $10 \mathrm{~m} \times 10 \mathrm{~m}$ & $100 \mathrm{~m} \times 50 \mathrm{~m}$ \\
House/Building height & $10 \mathrm{~m}$ & $7 \times 3 \mathrm{~m}$ \\
User density & $10-100 \mathrm{SUs} / \mathrm{km} 2$ & $1000-5000 \mathrm{SUs} / \mathrm{km} 2$ \\
Indoor-wall loss & $2 \mathrm{~dB}$ for TVWS, $4 \mathrm{~dB}$ for ISM & $6 \mathrm{~dB}$ for TVWS, $10 \mathrm{~dB}$ for ISM \\
Outdoor-wall loss & $4 \mathrm{~dB}$ for TVWS, $8 \mathrm{~dB}$ for ISM & $15 \mathrm{~dB}$ for TVWS, $20 \mathrm{~dB}$ for ISM \\
SU signal shadow fading & $\sigma=4 \mathrm{~dB}$ & $\sigma=6 \mathrm{~dB}$ \\
Noise level & $-174 \mathrm{dBm} / \mathrm{Hz}$ & $-174 \mathrm{dBm} / \mathrm{Hz}$ \\
\hline
\end{tabular}

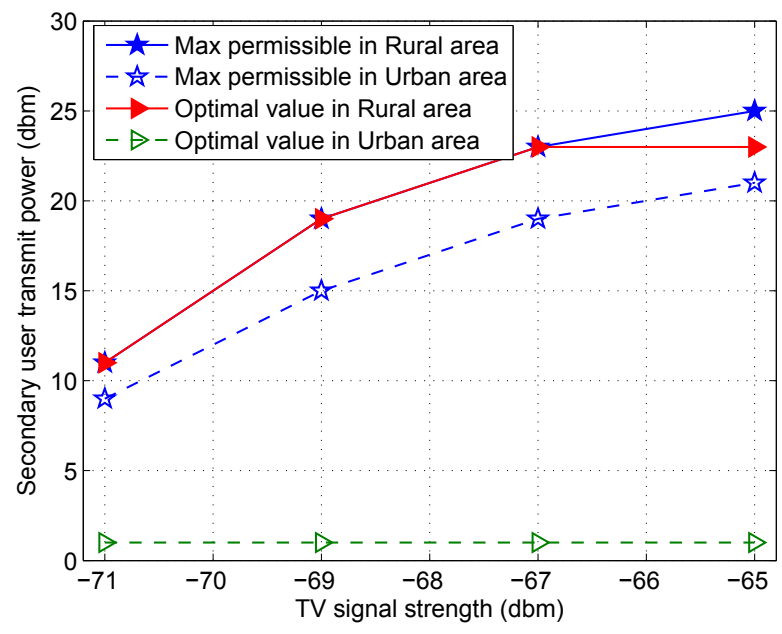

Fig. 4: Relation between permissible power and optimal power at different TV signal strength. $\lambda=30 S U s / \mathrm{km}^{2}$ in rural, $3000 \mathrm{SUs} / \mathrm{km}^{2}$ in urban, 2 channel aggregation, unoccupied $\mathrm{TV}$ channel number $=8$.

allows more active users to transmit simultaneously and causes a higher cumulative effect of multi-channel interference to the TV receiver. Therefore, the SUs are forced to reduce their transmit power and the throughput declines due to a lower SINR. Compared with the ISM band, around 16 unoccupied channels are required for TVWS system to achieve a higher throughput with aggregation of two TV channels, which is available in most European countries according to [6].

\section{Impact of User Density}

Fig. 7 depicts the maximum average throughput as a function of user density in both scenarios. Clearly, the decreasing lines illustrate that the Wi-Fi like system is more suitable for low density areas where collisions seldom happen. The increasing SU density would cause more collision avoidances as well as a lower permissible transmit power due to aggregate interference constraint. However, the shrinking gap between link throughput with different TV signal qualities in Fig. 7a

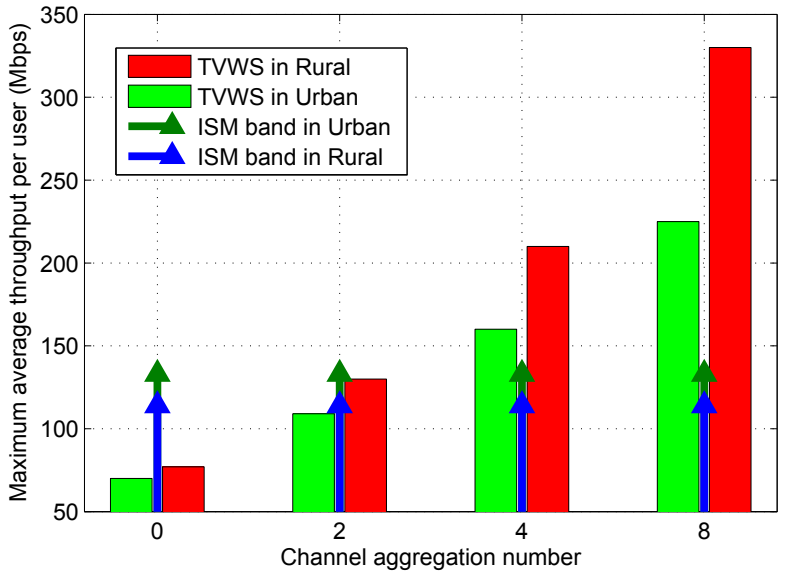

Fig. 5: Maximum average throughput versus channel aggregation scheme. $\lambda=30 \mathrm{SUs} / \mathrm{km}^{2}$ in rural, $3000 \mathrm{SUs} / \mathrm{km}^{2}$ in urban, TV signal strength $=-65 \mathrm{dBm}$, unoccupied TV channel number $=8$.

indicates that the network congestion has a stronger impact on the system performance than the limit on the permissible transmit power. The effect of potential collision is more obvious in the dense urban scenario, where traditional Wi-Fi in the ISM band, with a higher propagation loss and consequently less collision, outperforms the secondary system in TVWS.

\section{CONCLUSION}

In this paper, we have investigated the performance of a Wi-Fi like secondary network operating in TVWS with adaptive system configuration that can maximize its throughput while ensuring TV protection. Numerical evaluations have been performed for rural and urban environments, where the permissible secondary transmit power is adjusted according to the regulation frameworks for geo-location database assisted secondary system.

As indicated by simulation results, a secondary system with low density has a slight advantage over Wi-Fi in the ISM band due to the better coverage of the TV band, but it loses this advantage in the urban environment as the deployment density 


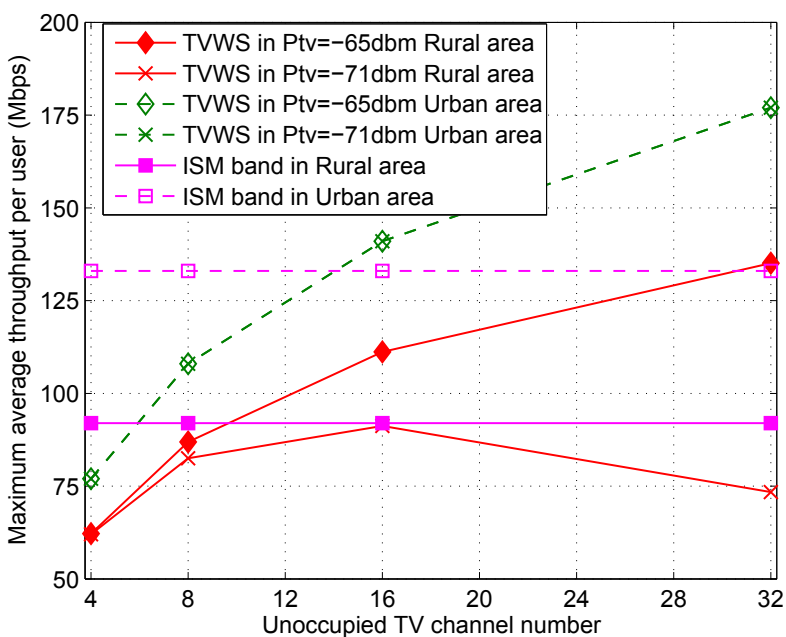

Fig. 6: Maximum average throughput versus unoccupied TV channel number. $\lambda=100 \mathrm{SUs} / \mathrm{km}^{2}$ in rural, $3000 \mathrm{SUs} / \mathrm{km}^{2}$ in urban, 2 channel aggregation.

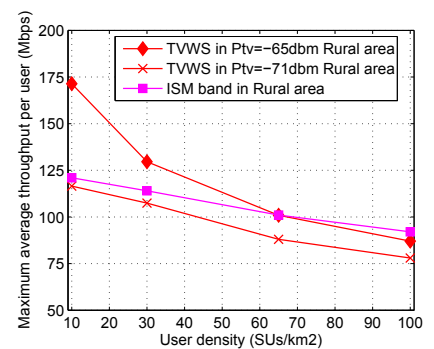

(a) Rural scenario

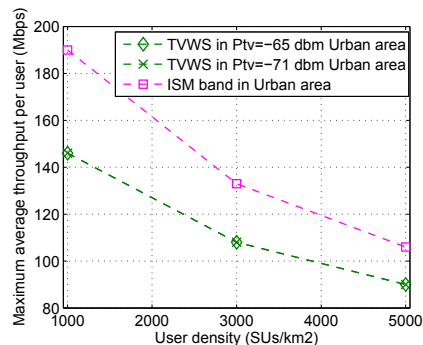

(b) Urban scenario
Fig. 7: Maximum average throughput versus user density, 2 channel aggregation, unoccupied TV channel number $=8$.

increases and the coverage distance shortens. The real strength of TVWS lies in its large amount of potentially available spectrum. With channel aggregation, the secondary system can easily outperform the Wi-Fi system in the ISM band. To our surprise, the interference constraint for TV protection only affects the achievable throughput of the secondary system in the rural scenario when the TV signal strength is extremely weak. Even though we have considered the cumulative effect of multi-channel interference and made pessimistic assumptions regarding the effect of secondary interference, in any other situations the capacity of the secondary system appears to be only limited by the secondary self-interference and the CSMA/CA protocol's attempt to avoid collision. These findings provide a deeper understanding on how the performance of a secondary system based on CSMA/CA is affected by the transmit power regulation in different environmental settings. It implies that the regulation framework should be more flexible in estimating the permissible transmit power and should distinguish the secondary systems with different MAC schemes, in order to achieve a highly efficient utilization of TVWS.

\section{ACKNOWLEDGMENT}

The research leading to these results has received partial funding from the European Union's Seventh Framework Programme FP7/2007-2013 under grant agreement $n^{\circ} 248303$ (QUASAR). The input and suggestions from the partners in the QUASAR project is gratefully acknowledged.

\section{REFERENCES}

[1] European Commission Report. (2012, Feb.) Perspectives on the Value of Shared Spectrum Access. [Online]. Available: http://ec.europa.eu/ information_society/policy/ecomm/radio_spectrum/sectorial/shared_use/

[2] M.Nekovee, "Quantifying the Availability of TV White Spaces for Cognitive Radio Operation in the UK," IEEE International Conference on Communications Workshops, June. 2009.

[3] FCC, "Second Memorandum Opinion and Order - In the Matter of Unlicensed Operation in TV Broadcast Bands Et Docket No.04-186Additional Spectrum for Unlicensed Devices Below 900Mhz and in The 3GHz Band ET Docket No.02-380," Sept. 2010.

[4] Ofcom. (2009, Nov.) Digital Dividend: Geolocation for Cognitive Access. [Online]. Available: http://stakeholder.ofcom.org.uk/consultations/ cogaccess/

[5] ECC Report 159. (2011, Jan) Technical and Operational Requirements for the Possible Operation of Cognitive Radio Systems in the TV White Spaces of the Frequency Band 470-790 MHz. [Online]. Available: http: $/ /$ www.ero.dk

[6] Jaap van de Beek, J. Riihijarve, A. Achtzehn and P. Mahonen, 'TV White Space in Europe," IEEE Transactions on Mobile Computing, Vol. 11, No. 2, Feb. 2012.

[7] B. Zayen, A. M. Hayar and D. Nussbaum, "Blind Spectrum Sensing for Cognitive Radio Based on Model Selection," in the 3rd International Conference on Cognitive Radio Oriented Wireless Networks Communications (CROWNCOM), May. 2008.

[8] H. R. Karimi, "Geolocation Databases for White Space Devices in the UHF TV Bands: Specification of Maximum Permitted Emission Levels," IEEE International Symposium On Dynamic Spectrum Access Networks (DySPAN), May. 2011.

[9] L. Shi, K. W. Sung and J. Zander, "Controlling Aggregate Interference Under Adjacent Channel Interference Constraint In TV White Space," in the 7th International Conference on Cognitive Radio Oriented Wireless Networks Communications (CROWNCOM), Jun. 2012.

[10] S. Kawade and M Nekovee, "Broadband Wireless Delivery Using An Inside-Out TV White Space Network Architecture," IEEE Global Telecommunications Conference (GLOBECOM), Dec. 2011.

[11] L. Simic, M. Petrova and P. Mahonen, "Wi-Fi, But Not on Steroids: Performance Analysis of a Wi-Fi-Like Network Operating in TVWS Under Realistic Conditions," IEEE International Conference on Communication, Jun. 2012.

[12] E. Obregon, L. Shi, J. Ferrer and J. Zander, "A model for aggregate adjacent channel interference in TV white space," in IEEE 73rd Vehicular Technology Conference (VTC), May. 2011

[13] L. Shi, K. W. Sung and J. Zander, "Secondary Spectrum Access in TVBands with Combined Co-Channel and Adjacent Channel Interference Constraints," IEEE International Symposium On Dynamic Spectrum Access Networks (DySPAN), Oct. 2012

[14] "Propagation data and prediction methods for the planning of short-range outdoor radio-communication systems and radio local area networks in the frequency range $300 \mathrm{MHz}$ to $100 \mathrm{GHz}$," ITU Recommendation P.1411.

[15] "Propagation data and prediction methods for the planning of indoor radio-communication systems and radio local area networks in the frequency range $900 \mathrm{MHz}$ to $100 \mathrm{GHz}$," ITU Recommendation P.1238.

[16] IEEE standard for Information technology - Telecomminications and information exchange between systems - Local and metroplitan area networks - Specific requirements - Part 11: Wireless LAN Medium Access Control (MAC) and Physical Layer (PHY) Specifications, Std. IEEE std 802.11-2007 Mar. 2011.

[17] "Directivity and Polarization Discrimination of Antennas in the Reception of Television Broadcasting," ITU-R Recommendation BT.419-3.

[18] Office for National Statistics. (2012, Jul.) 2011 Census - Population and Household Estimates for England and Wales. [Online]. Available: http://www.ons.gov.uk 